\title{
Competitividad, mercados del trabajo y estructura socioterritorial en Bogotá, Lima y Santiago ${ }^{1}$
}

\author{
Luis Fuentes ${ }^{2}$ y Felipe Link ${ }^{3}$
}

\begin{abstract}
RESUMEN
Las reformas económicas y laborales aplicadas con distinta intensidad y en distintos momentos en Chile, Colombia y Perú han incidido en el surgimiento de nuevos mecanismos de exclusión, que han contribuido a segmentar los mercados laborales y los espacios residenciales en Santiago, Bogotá y Lima. En este sentido el objetivo de este trabajo es intentar dar respuesta a las preguntas que surgen de este proceso: ¿cómo los procesos de liberalización económica y restructuración productiva que buscaron estimular el crecimiento económico de los países en el contexto de la globalización, han incidido en la transformación del mercado de trabajo urbano y en la estructura socioterritorial de estas ciudades? ¿La búsqueda por aumentar la competitividad es conciliable con una ciudad con menores niveles de exclusión urbana?
\end{abstract}

Palabras clave: Competitividad, mercados del trabajo, segregación.

\begin{abstract}
Economic and labor market reforms to varying degrees and at different times have affected the emergence of new mechanisms of exclusion in Chile, Colombia and Peru. These, in turn, have contributed to the segmentation of labor markets and residential developments in Santiago, Bogota and Lima. Thus the aim of this paper is to provide answers to questions that arise from this process: how have the processes of economic liberalization and productive restructuring, which sought to stimulate economic growth in these countries in the context of globalization, affected the transformation of the urban labor market and socio territorial structure of these cities? Is the search for increased competitiveness reconcilable with a city with lower levels of urban exclusion?
\end{abstract}

Keys words: Competition, Urban Labor Market, Socio spatial segregation.

\footnotetext{
Este trabajo fue realizado en el contexto del proyecto Fondecyt $N^{\circ} 11121477$, Fondecyt $N^{\circ} 11110354$ y CEDEUS, CONICYT/FONDAP No 15110020 Artículo recibido el 10 de diciembre de 2013, aceptado el 17 de abril de 2014 y corregido el 31 de julio de 2014.
}

\footnotetext{
2 Instituto de Estudios Urbanos y Territoriales, Pontificia Universidad Católica de Chile (Chile). E-mail: Ifuentes@uc.cl

3 Instituto de Estudios Urbanos y Territoriales, Pontificia Universidad Católica de Chile (Chile). E-mail: felipe.link@uc.cl
} 
El propósito de este trabajo es analizar, desde un enfoque comparativo, las consecuencias de las políticas de liberalización económica implementadas por Chile, Colombia y Perú desde la década de 1980 y 1990 respectivamente, en el contexto de la globalización, sobre dos de las más importantes dimensiones de la sociabilidad urbana, entendidas desde Kaztman (2001) como segmentación del mercado laboral y segregación residencial en Bogotá, Lima y Santiago.

La hipótesis que se pretende poner a prueba es que dadas las reformas económicas y laborales aplicadas en las últimas décadas, como parte de los procesos de reestructuración productiva impulsados por esos países, sus respectivas capitales se enfrentan a un proceso de erosión de sus patrones de convivencia, lo que obedece al surgimiento de mecanismos de exclusión que incrementan la segmentación de sus mercados del trabajo y la distribución de los distintos grupos sociales en el territorio metropolitano. Esto ha ocurrido pese a que estas reformas se produjeron en distintos momentos y con distintos grados de intensidad en cada uno de los países y que además cada área metropolitana cuenta con distintos modelos de administración y gestión de sus respectivos territorios.

El incremento en los niveles de segmentación en los mercados del trabajo y en el espacio urbano en las ciudades latinoamericanas han sido una de las principales preocupaciones de la investigación urbana. Algunos investigadores como Kaztman (2009), plantean que los procesos de transformación de las sociedades tradicionales a las sociedades modernas estuvieron marcados por la evolución de la solidaridad mecánica a la solidaridad orgánica, en donde los patrones de cohesión social enraizados en los lazos primarios fueron sustituidos por la universalización de los derechos de ciudadanía, en donde las ciudades han jugado un rol fundamental. Los factores causales de mayor erosión social actualmente están referidos a la segmentación en distintas esferas de la vida urbana: en el mercado del trabajo, en los servicios esenciales y en la distribución de la población en el territorio.

En el caso del marcado laboral urbano se analizan diversas estadísticas de las encuestas de empleo y del censo, datos con las que se construye un marco de evolución de las condiciones laborales de la población. En el segundo caso se analizan datos de los censos de población, se construyen distintas categorías socioocupacionales para el caso de Lima y Santiago y los niveles educativos en el caso de Bogotá y además, se aplican algunos índices de segregación tradicionalmente ocupados en la literatura, para analizar la segmentación socioespacial de las tres ciudades.

¿Por qué estas ciudades? La justificación que hace atractiva esta comparación obedece a sus similitudes, pero también a sus diferencias. Desde el punto de vista geográfico son las ciudades más importantes del Pacífico sur americano y la Comunidad Andina, localizadas en una de las zonas de comercio más importantes del mundo, como lo es el Foro de Cooperación Económica Asia Pacífico (CEAP); y en cuanto a su tamaño, se encuentran en un rango de población relativamente equivalente, 6 millones (Santiago), casi 7 millones (Bogotá) y 8,5 millones (Lima). Desde el punto de vista económico las tres economías nacionales aportan en torno a un $4 \%$ del PIB de la región, aunque existen importantes diferencias en el ingreso per cápita, siendo Chile el país con el mayor ingreso y crecimiento en el mediano plazo. En los últimos cinco años, las tres economías nacionales vienen creciendo a tasas superiores al 5\% anual, siendo la peruana la que alcanza el mejor promedio. Finalmente, desde el punto de vista político, Chile es una de las economías más abiertas del mundo, y Colombia y Perú han ido profundizando sus políticas de apertura, siendo los países que más han avanzado en el ranking de Libertad Económica de Heritage Fundation (HF) en los últimos años en América Latina, y junto con ello, han ido profundizando sus procesos de apertura externa con la firma de acuerdos de libre comercio.

Esta tendencia a profundizar sus estrategias de apertura externa y libertad económica, hace que sea interesante preguntarse sobre los efectos de estas políticas en las grandes áreas metropolitanas capitales, ya que estos lugares son los más expuestos a procesos de liberalización, apertura y modernización. Entonces las preguntas que se busca responder con el estudio comparativo son: ¿cómo la profundización de los procesos 
de liberalización económica y restructuración productiva que buscaron estimular el crecimiento económico de los países en el contexto de la globalización, han incidido en los mercados del trabajo y las estructuras socioespaciales?; ¿la búsqueda por competitividad influye en la segmentación del mercado laboral y en los patrones de segregación residencial?

\section{Reformas económicas y competitividad}

Brenner, Peck y Theodore (2009), plantean que el neoliberalismo se entiende de maneras muy diversas, como un conjunto de políticas, como un proceso tendencial de transformación institucional, como una forma emergente de la subjetividad, como un reflejo de los intereses hegemónicos reajustados, o como una combinación de estos. Algunos estudiosos ven estas tendencias como la señalización de una forma incipiente de convergencia normativa o hegemonía; otros continúan en llamar la atención sobre el flujo y la diversidad significativa, aunque aún no pueden determinar una contracorriente singular. Los más escépticos de tales visiones totalizadoras prefieren retratar el neoliberalismo como una forma híbrida de gubernamentalidad, o como una práctica reguladora dependiente del contexto.

Frente a todas estas aproximaciones $y$ tomando la idea del hibridismo, para el caso de este trabajo se entenderá de acuerdo a una definición de Harvey (2009), que el neoliberalismo es una teoría de prácticas políticoeconómicas que afirma que la mejor manera de promover el bienestar del ser humano consiste en no restringir el libre desarrollo de las capacidades y de las libertades empresariales del individuo dentro de un marco institucional caracterizado por los derechos de propiedad privada fuertes, mercados libres y libertad de comercio.

La evolución del neoliberalismo ha sido estudiada por varios autores, quienes han concluido que esta es específica, diversa y dificil de encerrar en una receta. Brenner, Peck y Theodore, plantean la noción de "neolibe- ralización híbrida ${ }^{4 \prime}$ para enfatizar el carácter constitutivamente incompleto, experimental y, finalmente, polimórfico de los procesos de neoliberalización, así como su carácter dependiente de la trayectoria de cada ola sucesiva de reestructuración regulatoria. El hecho de localizar el desigual desarrollo regulatorio en el centro de los procesos de neoliberalización, conceptual y empíricamente, proporciona una base para repensar su discontinuidad, ascendencia histórica ondulada, sus geografías diversas y polimórficas, sus contradicciones asociadas, sus tendencias a la crisis y sus límites contemporáneos.

En este sentido, tomando esta línea argumentativa se asume que los procesos de neoliberalización en América Latina, se dieron de diversas formas y bajo distintos contextos. Varias causas jugaron a favor de que poco a poco, casi todos los países de la región fueran adoptando un nuevo modelo de desarrollo basado en la apertura externa, desregulación y privatización, con el objetivo de hacer a sus países atractivos para la inversión internacional, en contextos y bajo particulares condiciones locales.

La aplicación de estas nuevas políticas orientadas a la competitividad en América Latina se dio con distinta intensidad y alcance, generalizándose a partir de finales de los ochenta y principios de los noventa. Así, desde el punto de vista de la temporalidad en su adopción, se podría clasificar a los países de la región en: reformistas prematuros o tem$\operatorname{pranos}^{5}$ a partir de mediados de los setentas (Chile), reformistas de primera oleada a partir de los ochentas (México y Bolivia), reformistas de segunda oleada a partir de finales de los ochenta y principios de los noventa

\footnotetext{
4 Traducido así de la expresión original: "Variegated neoliberalization"

5 En el primero de los grupos (primeros reformistas) Edwards (1997) incluye a Chile, México y Bolivia, sin embargo para el caso de esta investigación se estima necesario diferenciar a Chile del resto debido al temprano proceso de reformas vivido en el país, el cual se adelantó en al menos siete años a México y Bolivia. Además otros autores como Harvey, 2007 o Klein, 2007 han destacado las reformas realizadas en Chile como un verdadero experimento de los planteamientos de Milton Friedman, destacando lo temprano de su aplicación respecto a Estados Unidos (Reagan) y Reino Unido (Thatcher).
} 
(Argentina, Brasil, Colombia, Paraguay, Perú y Venezuela).

En Chile las reformas comenzaron en los meses que siguieron al golpe militar de $1973^{6}$, en donde se cambió radicalmente la política económica, abandonando de plano el antiguo modelo intervencionista. En Perú, la aplicación del modelo neoliberal también es intenso y comienza con la llegada de Alberto Fujimori al poder en 1990, quien aplica un estricto plan de ajuste estructural de la economía. El caso colombiano es distinto a los dos anteriores, ya que la aplicación del modelo fue mucho más gradual que los casos anteriores. Sin embargo los cambios más importantes se iniciaron a inicios de la década de los noventa, donde se ponen en marcha

Cuadro $\mathrm{N}^{\circ} 1$

Panorama general de las reformas estructurales en Chile, Colombia y Perú

\begin{tabular}{|c|c|c|c|c|c|}
\hline País & Reforma Fiscal & $\begin{array}{l}\text { Reforma } \\
\text { Comercial }\end{array}$ & $\begin{array}{c}\text { Reforma } \\
\text { Mercado } \\
\text { Financiero }\end{array}$ & $\begin{array}{c}\text { Reforma del } \\
\text { Mercado } \\
\text { Laboral }\end{array}$ & Privatización \\
\hline Chile & \begin{tabular}{|l|} 
Reforma \\
Fiscal, 1975 \\
presupuesto \\
equilibrado; \\
aumento de los \\
tipos fiscales; \\
en 1993, \\
modificación \\
de las leyes \\
fiscales de \\
1980
\end{tabular} & $\begin{array}{l}\text { Gran reforma } \\
\text { en 1975-1979; } \\
\text { restricciones } \\
\text { cuantitativas } \\
\text { eliminadas; } \\
\text { imposición } \\
\text { de aranceles } \\
\text { uniformes de } \\
\text { importación del } \\
11 \% \text {. }\end{array}$ & $\begin{array}{l}\text { Gran reforma } \\
\text { en 1975-1979; } \\
\text { tipos de interés } \\
\text { liberalizados; } \\
\text { sin asignación } \\
\text { de créditos; } \\
\text { cuenta de } \\
\text { capitales semi- } \\
\text { abierta }\end{array}$ & $\begin{array}{l}\text { Grandes } \\
\text { reformas en } \\
1979 ; \text { leyes } \\
\text { laborales } \\
\text { reformadas en } \\
1990\end{array}$ & $\begin{array}{l}\text { Importante, todas } \\
\text { las empresas } \\
\text { estatales } \\
\text { vendidas menos } \\
\text { 50; participación } \\
\text { total del sector } \\
\text { privado en } \\
\text { los servicios } \\
\text { públicos a } \\
\text { excepción del } \\
\text { agua hasta } 1994 .\end{array}$ \\
\hline Colombia & $\begin{array}{l}\text { Ajuste fiscal } \\
\text { iniciado, } \\
1985-1986 ; \\
\text { dos reformas } \\
\text { fiscales en } \\
1991 \text { y } 1992\end{array}$ & $\begin{array}{l}\text { Muy acelerada } \\
\text { en 1991; arancel } \\
\text { medio reducido } \\
\text { del } 83 \% \text { en } 1985 \\
\text { al 7\% en } 1992\end{array}$ & \begin{tabular}{|l|} 
Tipos de interés \\
liberalizados; \\
se mantiene \\
la asignación \\
de créditos; \\
imposición \\
de barreras de \\
acceso
\end{tabular} & $\begin{array}{l}\text { Reforma del } \\
\text { código laboral } \\
\text { en 1990; } \\
\text { permanecen } \\
\text { ciertas } \\
\text { distorsiones }\end{array}$ & $\begin{array}{l}\text { Escaso número } \\
\text { de empresas } \\
\text { estatales; } \\
\text { ninguna acción } \\
\text { significativa; } \\
\text { aranceles } \\
\text { redefinidos con } \\
\text { consideración de } \\
\text { los costos. }\end{array}$ \\
\hline Perú & $\begin{array}{l}\text { Sin reforma } \\
\text { fiscal; } \\
\text { mejora de la } \\
\text { administración } \\
\text { fiscal; control } \\
\text { sustancial del } \\
\text { gasto }\end{array}$ & $\begin{array}{l}\text { Inicio significativo } \\
\text { en 1990, } \\
\text { restricciones } \\
\text { cuantitativas } \\
\text { limitadas; arancel } \\
\text { del 5-15\%; } \\
\text { impuesto de } \\
\text { exportación } \\
\text { abolido; reforma } \\
\text { del sistema } \\
\text { aduanero }\end{array}$ & \begin{tabular}{|l|} 
En marcha, \\
bancos estatales \\
aún muy \\
importantes; \\
tipos de interés \\
libres, cierta \\
asignación de \\
créditos; cuenta \\
de capitales \\
abierta
\end{tabular} & $\begin{array}{l}\text { La } \\
\text { Constitución } \\
\text { de } 1993 \text { hace } \\
\text { más flexible } \\
\text { el mercado } \\
\text { laboral y } \\
\text { elimina } \\
\text { cláusulas de } \\
\text { estabilidad en } \\
\text { el empleo }\end{array}$ & $\begin{array}{l}\text { Gran acción } \\
\text { desde 1992; } \\
\text { el } 30 \% \text { de } \\
\text { las empresas } \\
\text { estatales ya } \\
\text { privatizadas } \\
\text { (incluida la } \\
\text { Compañía de } \\
\text { Hierro Perú) }\end{array}$ \\
\hline
\end{tabular}

Fuente: Extraído y modificado de Edwards, 1997.

\footnotetext{
6 Existe una discusión entre distintos autores acerca del comienzo de la implantación de las políticas neoliberales en Chile.
} 
las reformas estructurales, conocidas en el país cafetero como la "apertura económica". Estas reformas se emprendieron durante la administración Virgilio Barco (1986-1990), recibieron su mayor impulso a lo largo del gobierno de Gaviria (1990-1994) y se mantuvieron, con algunos matices, durante el gobierno de Ernesto Samper (Ocampo et al., 1998).

En el Cuadro $\mathrm{N}^{\circ} 1$, se presenta una mirada panorámica de las principales reformas realizadas en Chile, Colombia y Perú, países que con distinta intensidad, han insistido en aplicación del modelo hasta la fecha.

Tal como sostiene Edwards (1997), si bien la influencia del contexto mundial jugó un rol importante en la adopción de los nuevos postulados hacia el neoliberalismo, también hubo un consenso latinoamericano que estuvo influenciado de manera muy relevante por lo realizado en Chile de manera pionera en 1973. En este caso, nuevamente nos encontramos con importantes diferencias entre los procesos llevados a cabo por los países en cuestión. La primera y más evidente fue la temporalidad en la cual se realizaron las reformas, siendo Chile el país donde primero se adoptaron las reformas y 17 años después en Colombia y Perú. Otro de los rasgos distintivos es el contexto político y la rapidez de las reformas. En el caso de Chile y Perú, las reformas se realizaron en un contexto dictatorial y en forma de shock (Klein, 2007). Mientras que en Colombia se aplicaron en democracia y en forma bastante gradual. Las consecuencias de estas reformas estructurales pueden ser analizadas de una multiplicidad de formas, profundidad y en distintas escalas. En el caso de este apartado se intentará demostrar que uno de los principales efectos de las reformas realizadas en Chile, Colombia y Perú adscribiendo al modelo dominante y ha sido el fomento hacia la idea de convertir a sus territorios en lugares cada vez más atractivos y competitivos para los negocios internacionales.

Este nuevo modelo de desarrollo ha tenido como consecuencia un proceso de crecimiento sostenido de las economías ya que se ha manifestado en los casos de Chile y Perú con mayor evidencia, tal como lo muestra el Cuadro $\mathrm{N}^{\circ} 2$.

Cuadro $\mathrm{N}^{\circ} 2$

Crecimiento promedio anual del producto interno bruto de Chile, Colombia y Perú entre 1975 y 2008

\begin{tabular}{|l|rrrrrrr|}
\hline País & \multicolumn{7}{|c|}{ Crecimiento promedio anual del PIB (\%) } \\
\hline \multirow{3}{*}{ Chile } & $1975-1980$ & $1981-1985$ & $1986-1990$ & $1991-1995$ & $1996-2000$ & $2001-2005$ & $2006-2008$ \\
\cline { 2 - 8 } & 4,2 & $-0,5$ & 6,8 & 8,7 & 4,2 & 4,2 & 4,6 \\
Colombia & 4,9 & 2,3 & 4,6 & 4,6 & 1,0 & 3,9 & 7,2 \\
Perú & 2,7 & $-0,18$ & $-1,54$ & 5,72 & 2,52 & 4,21 & 8,30 \\
\hline
\end{tabular}

Fuente: Elaboración propia en base a datos de la CEPAL.

En Chile, salvo el quinquenio de principios de los ochenta, el país ha mantenido un ritmo de crecimiento constante sobre el $4 \%$, que tuvo su época de esplendor a principios de los noventa cuando el incremento del PIB superó el 8\% como promedio. En Perú ha pasado algo similar ya que posterior a las reformas realizadas en el año 1990 el crecimiento económico ha sido constante, sobre todo a partir de la última década y, en especial en los últimos años cuando alcanzó un crecimiento promedio superior al $8 \%$. Colombia es distinta a los anteriores que, antes de las reformas no hubo un proceso de crisis profunda como en Perú o en Chile y estas fueron realizadas paulatinamente. Esto se manifiesta en diferentes indicadores que han mejorado en la última década, y al igual que en Perú, de manera explosiva en los últimos años. 
Consecuencia de los avances en materia de crecimiento económico, Chile, Perú y Colombia han posicionado a sus ciudades principales en el mapa para los negocios dentro de la región latinoamericana, mejorando su atractivo.

Así lo demuestran las cifras del ranking de mejores ciudades para hacer negocios de la Revista América Economía, en donde se verifica que las ciudades de Bogotá y Lima han sido las que más han avanzado en el escalafón en los últimos años, conforme a un proceso de reformas económicas iniciado a principios de los noventa, pero que se ha ido consolidando a partir de la primera década del siglo XIX. Bogotá pasó del lugar trece al seis en el 2009 y en el ranking 2010 se ha posicionado en el lugar ocho. Mientras que Lima pasó del puesto veintitrés al séptimo lugar en el 2009 y se posicionó décima en la versión 2010. Santiago, fruto de un proceso de reformas económicas iniciado por Chile de manera mucho más temprana, desde la primera edición del ranking ya comenzó a tener un rol importante en el contexto latinoamericano, pasando del cuarto lugar al segundo en las últimas versiones.

Esta situación reafirma lo planteado anteriormente, mientras los países realizan reformas adaptándose, bajo ciertas particularidades, cada vez más al modelo predominante y logrando estabilidad política y macroeconómica, se transforman en lugares atractivos para la inversión nacional e internacional, siendo sus capitales o principales áreas metropolitanas, los lugares donde estos procesos se manifiestan con mayor energía.

\section{Precarización laboral y segmentación espacial en Bogotá, Lima y Santiago}

Desde la perspectiva neoliberal, antes de las reformas económicas, la mayor parte de las legislaciones laborales en Latinoamérica se caracterizaban por constituir un mercado excesivamente rígido, donde no solo resultaba difícil introducir nuevas tecnologías o modificar la orientación de las firmas sino que además los ajustes de mano de obra resultaban altamente complejos. Esto último, por los altos costos de los despidos, los engorrosos mecanismos para concretarlos y la existencia de monopolios en algunas actividades del mercado del trabajo (Coloma y Rojas, 2001).

Las reformas orientadas hacia un modelo de desarrollo económico de libre mercado, obligaron a generar un mercado del trabajo compatible con las nuevas exigencias de dinamismo y flexibilidad, lo cual "permitía reasignar recursos y permite que la economía responda rápidamente a los nuevos desafíos planteados por una mayor competencia exterior" (Edwards, 1997).

Para el caso específico de Chile, las regulaciones relativas al mercado del trabajo pueden ser divididas básicamente en dos períodos. El primero de 1974 a 1991 que tuvo como objetivo complementar el ajuste requerido por otras reformas estructurales e introducir flexibilidad en el mercado laboral, con el fin de promover una mayor eficiencia y reducir las altas tasas de desempleo que se elevaron hasta niveles de dos dígitos. En un segundo período, bajo los gobiernos democráticos después de 1991, se introdujeron nuevos cambios a la legislación laboral, los que contribuyeron a reforzar el poder negociador de los trabajadores, aunque de una manera coherente con el marco de mayor flexibilidad que se había impuesto en los años del gobierno militar (Coloma y Rojas, 2001).

Colombia, de manera mucho más tardía también registró importantes modificaciones a su regulación de los mercados del trabajo desde 1990. En un contexto democrático, las reformas fueron distintas a las chilenas, ya que combinó una flexibilización parcial del mercado laboral con una mayor protección de los derechos sindicales (Ocampo et al., 1998).

En el caso de Perú la desregulación del mercado laboral se inició en 1991 con la Ley de Fomento del Empleo. Esta ley introdujo un amplio conjunto de modalidades contractuales temporales y de plazo determinado y simplificó los procedimientos administrativos. También se permitió la creación de cooperativas de trabajadores y empresas de servicios que pudiesen proveer de trabajo a las empresas, es decir se plantea a la subcontratación como un mecanismo válido para proveer trabajo. Por otro lado, se introdujo mayor 
flexibilidad en las negociaciones entre empresas y trabajadores acerca de las jornadas de trabajo.

El supuesto básico con el que se aplicaron estas reformas en los tres países, es que liberar de estas distorsiones al mercado del trabajo permite tener efectos positivos en la distribución, por que estimula la expansión del empleo y aumenta los salarios en los segmentos más pobres de la sociedad (Edwards, 1997).

\section{La precarización del empleo}

Uno de los investigadores que más ha estudiado los mercados del trabajo latinoamericanos es Weller (2011). Este autor plantea que las reformas de flexibilización han producido una serie de efectos en Amérca Latina. En el caso de este trabajo nos concentraremos en la tendencia hacia la precarización de las condiciones laborales teniendo como hipótesis que se están acrecentando las segmentaciones en el acceso al empleo de calidad en los mercados del trabajo metropolitanos en estudio. Para esto se analiza diversa información proveniente de fuentes oficiales, principalmente encuestas de empleo y hogares de los respectivos países. Como limitantes está el hecho de que no se cuenta con exactamente la misma información para las tres ciudades, lo cual limita la comparación, sin embargo se intentará mostrar evidencia estadística, en algunos casos parcial, para poner a prueba estos tipos de exclusiones que se estarían acrecentando, según se plantea en la bibliografía especializada.

Entonces respecto al acceso al empleo de calidad, en el caso de Santiago, los datos de la encuesta CASEN muestran que en general el empleo precario se ha expandido. En 1990 el promedio de asalariados sin contrato Ilegaba al $15,2 \%$ y esa cifra se ha expandido a $19,5 \%$ en el 2009 . Por género, las mujeres asalariadas en promedio al año 2009 tienen un $10 \%$ menos de acceso al empleo protegido por contratos. Si analizamos los datos considerando los grupos etarios, el grupo en donde más ha crecido el empleo desprotegido ha sido en los más jóvenes, en donde en 1990 un $27,1 \%$ trabajaba sin contrato y esa cifra en el 2009 se expandió al 36,2\%, siendo el grupo más perjudicado, ya que el resto se encuentran en torno al promedio o por debajo de él. Ahora si analizamos la misma variable pero cruzada por ingresos nos encontramos con un panorama similar, tal como se observa en el Cuadro $\mathrm{N}^{\circ} 3$ en donde existe una relación proporcional entre pobreza y acceso al trabajo sin contrato. En general se observa que en todos los deciles crece el empleo desprotegido, sin embargo la proporción de asalariados del decil más pobre prácticamente triplica al decil más rico en ambos períodos. Además el decil en el que más crece el empleo desprotegido es en el decil más pobre, sin embargo la brecha respecto al resto no es tan amplia como los casos anteriores.

Cuadro $\mathrm{N}^{\circ} 3$

Asalariados sin contrato de trabajo por decil de ingresos en RMS

\begin{tabular}{|r|r|r|r|}
\hline $\begin{array}{c}\text { Decil de } \\
\text { ingresos }\end{array}$ & 1990 & 2009 & Variación \\
\hline 1 & 24,7 & 32,4 & 7,7 \\
2 & 17,3 & 22,8 & 5,5 \\
3 & 16,1 & 20,5 & 4,4 \\
4 & 17,3 & 19,6 & 2,3 \\
5 & 13,1 & 15,1 & 2,0 \\
6 & 13,1 & 16,8 & 3,7 \\
7 & 11,4 & 12,9 & 1,5 \\
8 & 11,8 & 12,7 & 0,9 \\
9 & 7,0 & 12,3 & 5,3 \\
10 & 8,3 & 12,5 & 4,2 \\
\hline
\end{tabular}

Fuente: Elaboración propia en base a datos de la Encuesta Casen, diversos años.

Si bien en Chile el empleo informal es uno de los más bajos de Latinoamérica, distintos estudios plantean que no es un tema poco relevante ya que por ejemplo, según las cifras de la CEPAL habría bajado desde 38\% a $30 \%$ entre 1990 a 2009, están cifras sin dudas son más altas que las que existían en 1978 antes de las reformas laborales, las que alcanzaban el 25,3\% (Fuentes \& Meneses, 1987). Esto es preocupante ya que en este caso a la desprotección laboral, se suma la desprotección en materia de salud y previsión social, lo cual implica un aumento en el grado de riesgo e inseguridad para un número importante de familias. También algunos 
estudios realizados han puesto el acento en el alto nivel de fragilidad en la duración del empleo. Por ejemplo se estima según cifras del Instituto Nacional de Estadísticas que el $41 \%$ de los empleos en el sector privado dura menos de un año.

Para Lima metropolitana, la información contenida en el siguiente cuadro muestra el mismo fenómeno descrito para Santiago, es decir una expansión del empleo sin contrato, el cual ya en 2001 era bastante alto y aumenta aún más al 2007. El empleo con contrato indefinido disminuye fuertemente en $16 \%$. Junto con el empleo sin contrato también se expande el contrato a corto plazo y a honorarios profesionales y todas modalidades ligadas a los procesos de subcontratación que se han hecho masivos bajo las nuevas formas de gestión de las grandes empresas y el Estado.

Cuadro $\mathrm{N}^{\circ} 4$

Evolución de las modalidades de contratación en Lima Metropolitana

\begin{tabular}{|l|r|r|r|r|}
\hline Modalidad & \multicolumn{1}{|c|}{1992} & \multicolumn{1}{|c|}{2001} & \multicolumn{1}{|c|}{2007} & \multicolumn{1}{c|}{ Variación } \\
\hline Indefinido & 35,3 & 30,1 & 19,3 & -16 \\
corto plazo & 18,4 & 11,6 & 20,9 & 2,5 \\
Comisión & 8,2 & 5,8 & - & - \\
Cooperativas & - & 4,1 & - & - \\
Honorarios profesionales & - & 3,9 & 10,6 & - \\
Sin contrato - sin beneficios & 37,7 & 44,5 & 48,7 & 11 \\
Otros & 0,2 & 0 & 0,5 & 0,3 \\
Total & & 100 & 100 & - \\
\hline
\end{tabular}

Fuente: MTPE - Programa de Estadísticas y Estudios Laborales.

Estas nuevas modalidades de contratación han tenido como consecuencia una alta rotación y menor duración del empleo, tal como lo ha advertido el Ministerio de Trabajo y Promoción del Empleo del Perú (2002) a través de las encuestas realizadas periódicamente. Por ejemplo en 1991 la duración media de los empleos privados en Lima Metropolitana era de seis años, la cual disminuyó a cuatro en 2001 .

Cuando se analiza y se cruza esta variable por sexo, edad y grupos ocupacionales se observan importantes brechas. Por ejemplo entre 1996 y 2001 existe una importante diferencia entre la duración de los empleos de los hombres y las mujeres, en el caso de los primeros se pasa de 5,2 a 3,9 años y en el caso de las mujeres de 3,2 a 2,6 años promedio. En cuanto a la variable edad, los segmentos más jóvenes tienen una duración promedio del empleo mucho más bajo que los segmentos de mayor edad (1,3 años en
1996 a 1 año en 2001 en el caso de los más jóvenes y 11,8 a 9,8 años en el segmento más adulto). Al mismo tiempo se observa que la tendencia a la mayor movilidad es común a todos los grupos de edad.

Por grupos ocupacionales, se observan importantes diferencias en la duración promedio de los empleos, ya que mientras en el nivel directivo se tenía una duración de casi nueve años en los empleos, los no calificados no superaban los cuatro años. Aunque todos los grupos disminuyen la duración del empleo, los grupos de menor calificación alcanzan altos niveles de movilidad, lo cual según el mismo Ministerio de Trabajo y Promoción del Empleo (2002) es perjudicial tanto para las empresas, pero por sobre todo para los trabajadores. Las principales consecuencias de este fenómeno para las personas es el aumento de la inseguridad en los ingresos, la disminución de posibilidades de capacitación al interior de las empresas, intermitencia en 
el aporte a las pensiones y exposición a la falta de cobertura de salud, entre otros.

Estas tendencias, no han hecho otra cosa que reforzar la informalidad, la cual ya era muy alta antes del comienzo de las reformas al mercado del trabajo, aunque se equiparaba al mercado formal. Sin embargo a partir de los noventa se expande de manera importante alcanzando niveles en algunos años superiores al $60 \%$, para terminar en el 2009 en un $59,8 \%$.

Llama la atención esta situación ya que a pesar del importante crecimiento económico del Perú durante los noventa, el empleo informal no ha logrado reducirse y persiste como el motor de la economía limeña. Los estudios realizados por el Ministerio del Trabajo y Promoción del Empleo, cruzando estos datos de acuerdo a distintas características, como la instrucción y las ocupaciones llegan a la conclusión del carácter estructural de la informalidad en el Perú y su permanencia en el tiempo a pesar del período de alto crecimiento económico, en el cual, el mercado formal no ha logrado absorber más empleos.

Para Bogotá, no fue posible establecer la evolución del empleo de acuerdo a las modalidades de contratación, ya que solo se obtuvo el dato del año 2008, el cual se puede observar en el Cuadro $N^{\circ} 5$. En él se observa la alta proporción de ocupados no asalariados o sin información, con los cuales se tiene una alta probabilidad de que no tengan ningún vínculo formal con su empleo, a lo cual si se les suma los ocupados con contrato, la cifra supera el $50 \%$ en ambos sexos.

\section{Cuadro $\mathrm{N}^{\circ} 5$}

Población ocupada según tipo de contrato en Bogotá

\begin{tabular}{|l|r|r|}
\hline & Hombre & \multicolumn{1}{|c|}{ Mujer } \\
\hline Contrato fijo & 11 & 10,9 \\
Contrato indefinido & 31,1 & 31,1 \\
Sin contrato & 12,6 & 9,9 \\
Sin información o no & 45,2 & 47,8 \\
asalariado & & \\
\hline
\end{tabular}

Fuente: Pineda, 2010.

En cuanto a la estabilidad del empleo, los datos recopilados por la Secretaría Distrital de
Planeación de Bogotá muestran que la duración ha venido cayendo con el avance del tiempo, al menos en la última década. Por ejemplo en el caso de los jóvenes, la duración promedio del empleo en el 2005 era de 0,7 año, la cual disminuyó a solo 0,3 en el 2007. En general, un $28,7 \%$ de los asalariados permanece un año en el trabajo, un 12,9 dura como promedio dos años y un 9,9 dura tres años. Solo un 33,4\% de los asalariados dura más de 6 años en el trabajo como promedio. Como en los casos anteriores, los jóvenes son los más afectados por esta inestabilidad del empleo.

Al igual que en Lima, la capital colombiana se caracteriza por el alto nivel de informalidad de su economía. Las regulaciones introducidas en los mercados del trabajo y los períodos de crecimiento económico experimentados por la capital colombiana no han tenido efecto en formalizar a la fuerza de trabajo. Desde el año 1992 ya era alta, lo cual se ha acentuado más aún, Ilegando a casi $53 \%$ en el 2005. La situación por género también queda de manifiesto, en donde se observa que las mujeres están más expuestas al mercado informal que los hombres. La informalidad parece ser un fenómeno estructural de la economía bogotana, ya que cruzada por distintas variables como género, posición ocupacional, los niveles de instrucción, entre otros, el fenómeno se hace persistente.

Las tendencias encontradas en las tres ciudades apuntan al aumento del empleo de mala calidad que se manifiesta en la disminución de los empleos con contrato, el aumento en la movilidad y consecuente disminución de la duración del empleo y el aumento en la informalidad. Esta situación es preocupante en la medida en que la informalidad se caracteriza por la desprotección en materia de salud y seguridad social, lo cual sumado a la retirada del Estado de la vida social de las personas crea un panorama complejo sobre todo para los sectores que se ven más afectados por esta tendencia.

\section{Segmentación socioespacial en Bogotá, Lima y Santiago}

La discusión contemporánea en torno a la evolución de la metrópolis en la era de la globalización se ha centrado fuertemente en la adscripción o no adscripción a las caracte- 
rísticas propias de la ciudad global descritas por Sassen hace más de veinte años. Es decir, la discusión se ha centrado en comprobar o refutar ciertas tendencias hacia una polarización social y espacial en las ciudades que sufren las transformaciones más radicales de la reestructuración productiva posfordista, que Sassen (1991) identifica en Londres, Nueva York y Tokio. Sin embargo, a pesar del inevitable e interesante debate entre quienes confirman esta tendencia y quienes la refutan, aludiendo por ejemplo a un crecimiento de los sectores medios de la sociedad y a una profesionalización de la población, más que a la polarización (Hamnett, 1996), pareciera que las indiscutibles transformaciones urbanas y de la sociedad en la globalización, solo tengan como horizonte de posibilidad en el discurso académico, una dicotomía entre dualización o medianización homogénea. En otras palabras, entre la fractura en dos o el conjunto uniforme sin mayores tensiones. En este contexto, si bien entendemos como tendencia general, un proceso de medianización de la estructura social en las ciudades analizadas, este proceso tiene una serie de elementos de contexto que complejizan el análisis.

Por lo tanto, respecto a las estructuras socioespaciales de las ciudades es posible afirmar que las tres ciudades presentan un marcado sector en donde se concentran los grupos altos de la pirámide social, tal como se observa en la Figura $\mathrm{N}^{0} 1$. En el caso de Bogotá es del centro histórico al norte, en el caso de Lima en el centro geográfico de cerro a costa y en el caso de Santiago del centro histórico hacia el oriente. Sin embargo a pesar de esta similitud, la estructura social de este sector tradicional de altos ingresos es diferente en las ciudades. En el caso de Bogotá y Santiago existe mayor diversidad social, ya que otros grupos de una clase social más baja también tienen presencia entre ellos, en Lima es mucho más homogéneo y solo se comparte espacio con grupos medios principalmente.

Por el contrario, la localización de los sectores más bajos de la pirámide social queda relegada a las zonas más periféricas de la ciudad, en el caso de Lima en los bordes externos de los conos, en el caso de Santiago en el poniente y sur de la ciudad formando una especie de media luna, y en el caso de Bogotá principalmente en el sector sur de la ciudad, en todos los casos lejos de los centros y nuevos desarrollos.

El estudio de la distribución de la población en base a los índices sintéticos de segre-

Figura $\mathrm{N}^{\circ} 1$

Sectores de altos ingresos en Bogotá, Lima y Santiago en 2005, 2007 y 2002, respectivamente

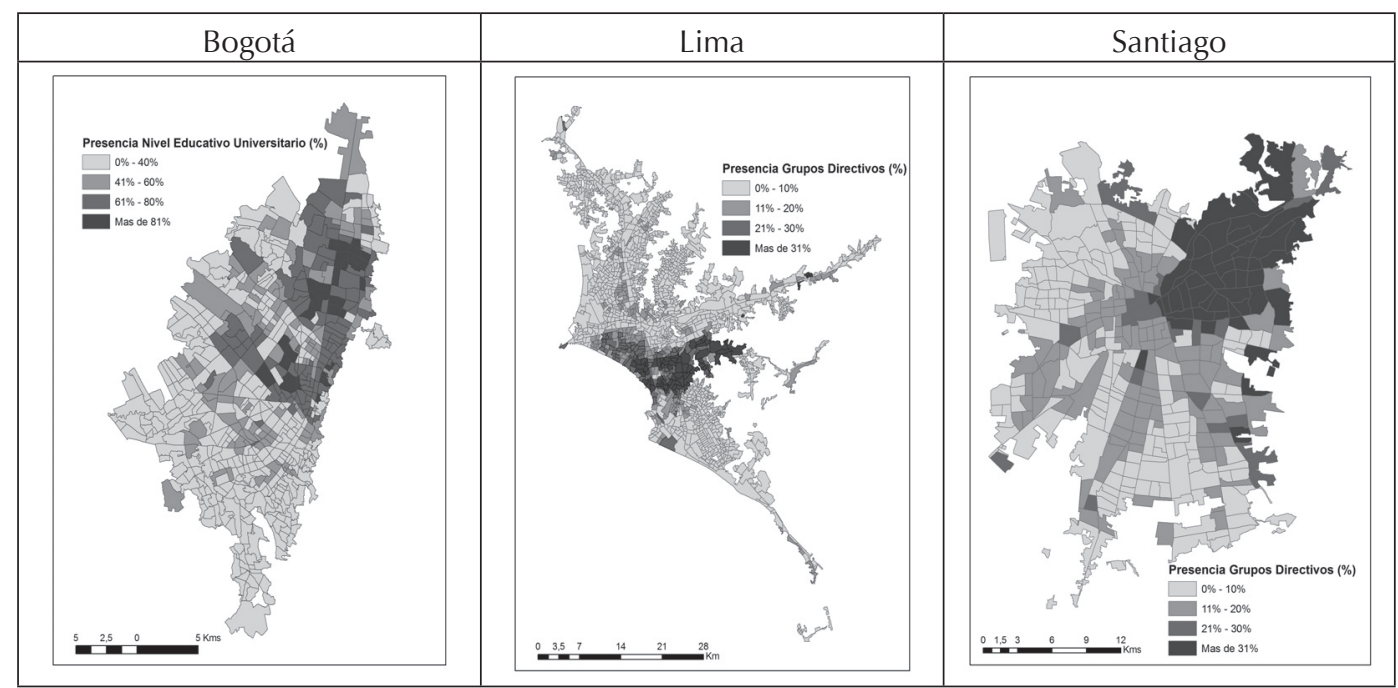

Fuente: Elaboración propia en procesamiento de información censal. 
gación ha sido ampliamente utilizado. De hecho, algunos autores como Sabatini, Cáceres y Cerda (2001), han replanteado y adaptado al caso latinoamericano la propuesta inicial planteada por Massey y Denton (1988) para las ciudades norteamericanas. Este autor plantea que para el caso de Latinoamérica la segregación consta de tres dimensiones: la tendencia de los grupos sociales a concentrarse en algunas áreas de la ciudad, la conformación de áreas o barrios socialmente homogéneos, y la percepción subjetiva que los residentes tienen de la segregación "objetiva". Para el caso de esta investigación se utilizarán las primeras dos dimensiones, las cuales son objetivas y se ajustan a las necesidades para entender y comparar el comportamiento de las dinámicas socioespaciales y la evolución de las estructuras metropolitanas. De esta manera para realizar una caracterización de las tendencias de segregación se considerarán los índices de disimilaridad de Duncan para analizar los grados de concentración de los grupos en el espacio y el índice de aislamiento o exposición para analizar la homogeneidad socioocupacional de un territorio (Duncan \& Duncan, 1955; Katzman, 2009; Sabatini, 2004; Sabatini et al., 2008; Sabatini y Sierralta, 2006; Rodríguez, 2001; entre otros), siguiendo una parte de la tradición de los estudios sobre segregación residencial en Latinoamérica.

Como variable de estratificación social se utilizó a los grupos socioocupacionales para Lima y Santiago y los niveles educativos para el caso de Bogotá7. Respecto a la escala

\footnotetext{
7 Las categorías socioocupacionales son elaboradas preferentemente en base a la variable categoría ocupacional del censo de los diferentes países, que se rige por la definición de la Clasificación Internacional Uniforme de Ocupaciones (CIUO) de 1988, la cual tiene como objetivo definir funciones $y$ tareas que desempeñan las personas en sus trabajos y situar estos en una perspectiva internacional comparable. Como en el caso del censo de Colombia no existe la pregunta que permite determinar la categoría ocupacional de cada individuo se utilizó como proxy el nivel educacional. La justificación para utilizar esta información está en las correlaciones realizadas para el caso de Santiago en donde se observa que existe una estrecha relación entre las categorías y las competencias educacionales. Tal comprobación también ha sido revisada en documentos de ChileCalifica (2005), al intentar caracterizar el mercado laboral de la RM (ChileCalifica, 2005).
}

de trabajo en el caso de Bogotá se utilizó el sector censal, en Lima las zonas censales y en Santiago los distritos censales, todas escalas relativamente comparables y equivalentes en superficie. La pregunta que se plantea en este apartado es si existe mayor segmentación de los espacios residenciales de las tres áreas metropolitanas, lo esperable sería que los índices de segregación mostraran un mayor grado de concentración y homogeneidad. Esto implicaría no solamente una diferenciación social de los distintos barrios sino también barreras de entrada, en el caso de los grupos de elite y de salida en los grupos más vulnerables.

Respecto a la evolución de la estructura social de las ciudades, esta se puede apreciar en el Cuadro $\mathrm{N}^{\circ} 6$. En cuanto a las comparaciones entre las tres ciudades, es importante considerar que se está en presencia de áreas metropolitanas con distintas características desde el punto de vista de su estratificación socioocupacional y educacional.

En cuanto a las diferencias entre las ciudades es interesante poner en relieve las diferencias en las tendencias de los grupos directivos y de los obreros en Lima y Santiago, los cuales se comportan de una manera distinta. Respecto a las similitudes, la tendencia común a las tres ciudades sería la aparición de un proceso de medianización que estarían experimentando las ciudades, donde los grupos medios y los trabajadores de comercio y servicios, crecen con fuerza, pasando a ser los grupos mayoritarios de la estratificación social. Esta tendencia cuestiona aquellas hipótesis vinculadas a la dualización urbana de las ciudades en proceso de globalización. Sin embargo, también hay que tener presente que el proceso de medianización en este sentido, corresponde muchas veces a un cambio de categoría ocupacional, sin estar vinculado necesariamente a una mejor situación socioeconómica o a una mejor posición social, dadas las características de inestabilidad y precarización del empleo contemporáneo (Link, 2013). Por lo tanto, el propio proceso de medianización descrito puede esconder muchas veces un incremento de la desigualdad socioterritorial, asociada a la microfragmentación urbana y a la polarización social en algunos sectores de la ciudad (Link, 2010). 


\section{Cuadro $\mathrm{N}^{\circ} 6$}

Estructura y variación porcentual de la participación de las categorías socio ocupacionales y educacionales para Bogotá, Lima y Santiago

\begin{tabular}{|c|c|c|c|c|c|c|c|c|}
\hline \multicolumn{2}{|l|}{ Categorías } & \multicolumn{2}{|c|}{ Bogotá } & \multicolumn{2}{|c|}{ Lima } & \multicolumn{3}{|c|}{ Santiago } \\
\hline & & $\begin{array}{c}\text { Estructura } \\
2005 \\
(\%)\end{array}$ & $\begin{array}{c}\text { Variación } \\
1993- \\
2005(\%)\end{array}$ & $\begin{array}{c}\text { Estructura } \\
2007 \\
(\%)\end{array}$ & $\begin{array}{c}\text { Variación } \\
1993- \\
2007 \text { (\%) }\end{array}$ & $\begin{array}{c}\text { Estructura } \\
2002 \\
(\%)\end{array}$ & $\begin{array}{c}\text { Variación } \\
1982- \\
1992 \text { (\%) }\end{array}$ & $\begin{array}{c}\text { Variación } \\
1992- \\
2002 \\
(\%)\end{array}$ \\
\hline Directivos & $\begin{array}{l}\text { Superior y } \\
\text { posgrado }\end{array}$ & 32,5 & 9 & 8,2 & $-3,8$ & 14,8 & 36,6 & 32,8 \\
\hline Medios & & 46,2 & 48,5 & 25,1 & 107,2 & 31,8 & 103,9 & 43,3 \\
\hline $\begin{array}{l}\text { Comercio } \\
\text { y servicios }\end{array}$ & Secundaria & & & 21 & 130,2 & 14,2 & 33,6 & 48,5 \\
\hline Obreros & $\begin{array}{l}\text { Preescolar } \\
\text { y primaria }\end{array}$ & 20,2 & 14 & 25,6 & 70,7 & 20,5 & 7,8 & $-14,6$ \\
\hline $\begin{array}{l}\text { No } \\
\text { calificados }\end{array}$ & Sin & 1,1 & 52,6 & 19,5 & 26,2 & 17,8 & 65,7 & 12,8 \\
\hline Agrícolas & & & & 0,6 & 2 & 1 & $-23,5$ & $-13,5$ \\
\hline
\end{tabular}

Fuente: Elaboración propia en base a información de los censos de población. Para Bogotá la variación es entre 1993 y 2005, para Lima entre 1993 y 2007 y para Santiago entre 1982 y 2002.

Una vez conocidas las tendencias de la evolución de la estratificación socioocupacional en cuanto a las cifras y representatividad, lo importante ahora es saber cómo estos distintos grupos se distribuyen espacialmente y cuáles han sido los patrones de localización. ¿Qué tan segregados están cada uno de estos grupos en el espacio metropolitano? ¿Cuáles son las tendencias comunes y los principales cambios experimentados? ¿Están más segmentados los espacios residenciales de las ciudades actuales?

El Cuadro $N^{\circ} 7$ muestra una situación comparativa respecto a las tendencias de segregación de las tres áreas metropolitanas. Los valores indican el valor de Duncan ${ }^{8}$ obtenido para los años evaluados. Realizando

\footnotetext{
8 El índice de Duncan mide la uniformidad en que están distribuidos los grupos sociales, en este caso los grupos socioocupacionales. El valor cercano a 0 indica que la distribución de la población de esa categoría es similar a la media de la distribución en la ciudad. Un valor cercano a 1 indicaría máxima segregación, donde no hay mezcla de grupos socioocupacionales o educacionales. El valor indica el porcentaje de población que debería redistribuirse
}

un análisis por categoría se observa que los directivos y educación superior, componen el grupo más segregado. Las tendencias son diversas ya que mientras en Bogotá y Santiago prácticamente se mantiene estable su concentración, en Lima aumenta. Los grupos medios poseen niveles de concentración semejantes en las tres ciudades. Respecto al comportamiento de este grupo, la tendencia común es al aumento de la concentración espacial, siendo Bogotá donde el fenómeno se daría con mayor fuerza. Respecto al grupo de trabajadores de comercio y servicio, que en el caso de Bogotá entraría en la misma categoría, poseen grados de segregación más bajos en todas las ciudades, en Lima su concentración disminuye y en Santiago aumenta levemente. 
Índices de disimilaridad de Duncan para Bogotá, Lima y Santiago por categorías

\begin{tabular}{|c|c|c|c|c|c|c|c|c|}
\hline \multicolumn{2}{|l|}{ Categorías } & \multicolumn{2}{|c|}{ Bogotá } & \multicolumn{2}{|c|}{ Lima } & \multicolumn{3}{|c|}{ Santiago } \\
\hline & Superior y & 1993 & 2005 & 1993 & 2007 & 1982 & 1992 & 2002 \\
\hline Directivos & posgrado & 0,45 & 0,44 & 0,35 & 0,44 & 0,37 & 0,34 & 0,37 \\
\hline Medios & & & & 0,23 & 0,24 & 0,18 & 0,21 & 0,19 \\
\hline $\begin{array}{l}\text { Comercio y } \\
\text { servicios }\end{array}$ & Secundaria & 0,18 & 0,21 & 0,12 & 0,11 & 0,09 & 0,11 & 0,11 \\
\hline Obreros & $\begin{array}{l}\text { Preescolar y } \\
\text { primaria }\end{array}$ & 0,30 & 0,30 & 0,25 & 0,23 & 0,30 & 0,26 & 0,24 \\
\hline No calificados & Sin & 0,28 & 0,32 & 0,18 & 0,16 & 0,15 & 0,16 & 0,20 \\
\hline Agrícolas & educación & & & 0,45 & 0,42 & 0,40 & 0,35 & 0,29 \\
\hline
\end{tabular}

Fuente: Elaboración propia en base a información de los censos de población. Para Bogotá la variación es entre 1993 y 2005, para Lima entre 1993 y 2007 y para Santiago entre 1982 y 2002.

Respecto a los trabajadores obreros, tanto en Lima como en Santiago, constituyen el segundo grupo más segregado (sin considerar los agrícolas) y el equivalente educacional para Bogotá también concuerda con esta tendencia y respecto a la variación en el período analizado, en Bogotá mantienen sus grados de concentración espacial, mientras que en Lima y Santiago disminuyen en un valor equivalente. Respecto a los trabajadores no calificados, en Santiago y Lima, no poseen valores tan altos de concentración espacial, y respecto a sus tendencias, mientras en Lima disminuye, en Santiago y su equivalente educacional en Bogotá aumenta.

La segunda dimensión de la segregación, que puede denominarse por exclusión, atañe estrictamente a la ausencia de mezcla o integración de grupos sociales en espacios comunes. En esta dimensión de la segregación, un grupo social no se mezcla con el resto aunque esté diseminado en varias partes de la ciudad. Así, se originan zonas homogéneas en un contexto heterogéneo, lo que probablemente dificulta la interacción (o encuentro al menos) con otros grupos sociales" (Rodríguez, 2001). Según plantean varias investigaciones, esta sería la dimensión maligna de la segregación (Katzman, 2009; Sabatini et al., 2008; entre otros), debido a sus consecuencias en la formación de guetos pobres y la proliferación de patologías sociales, como el desempleo, drogadicción y economía del delito, entre otros.

El Cuadro $N^{\circ} 8$ nos muestra un resumen de la composición social de los barrios de las ciudades en estudio, así como las tendencias respecto al aislamiento ${ }^{9}$ de los distintos grupos. Se observa que las ciudades en análisis poseen estructuras socioespaciales similares, ya que por ejemplo Lima y Santiago los grupos más aislados son los obreros, seguidos por los trabajadores medios, directivos y no calificados.

En ambos casos, los que aparecen menos aislados son los trabajadores del comercio y los servicios, los que en general habitan barrios diversos. Estos resultados contradicen la creencia común, en la cual se piensa que la elite es la que posee los grados más importantes de segregación, lo cual no se cumple en estas dos ciudades.

\footnotetext{
9 Para medir esta dimensión de la segregación para el caso de este estudio se utilizó el índice de interacción o aislamiento, el cual mide "el grado en que los miembros de un grupo $\mathrm{X}$ están expuestos al grupo $\mathrm{Y}$ en las diferentes subunidades territoriales. "Su interpretación indica, por ejemplo, que si su valor es 0.2 , en promedio en una unidad donde reside un miembro del grupo $X$, dos individuos de cada diez son del grupo" (Katzman, 2009:35)
} 
Cuadro $\mathrm{N}^{\circ} 8$

Índices de aislamiento para Bogotá, Lima y Santiago por categorías

\begin{tabular}{|c|c|c|c|c|c|c|c|c|}
\hline \multicolumn{2}{|l|}{ Categorías } & \multicolumn{2}{|c|}{ Bogotá } & \multicolumn{2}{|c|}{ Lima } & \multicolumn{3}{|c|}{ Santiago } \\
\hline & & 1993 & 2005 & 1993 & 2007 & 1982 & 1992 & 2002 \\
\hline Directivos & $\begin{array}{l}\text { superior y } \\
\text { posgrado }\end{array}$ & 0,42 & 0,49 & 0,23 & 0,18 & 0,23 & 0,22 & 0,25 \\
\hline Medios & & & & 0,27 & 0,30 & 0,21 & 0,30 & 0,35 \\
\hline $\begin{array}{l}\text { Comercio y } \\
\text { servicios }\end{array}$ & Secundaria & 0,50 & 0,50 & 0,17 & 0,22 & 0,13 & 0,12 & 0,15 \\
\hline Obreros & $\begin{array}{l}\text { Preescolar y } \\
\text { primaria }\end{array}$ & 0,34 & 0,27 & 0,32 & 0,30 & 0,45 & 0,35 & 0,25 \\
\hline $\begin{array}{l}\text { No } \\
\text { calificados }\end{array}$ & Sin educación & 0,02 & 0,02 & 0,22 & 0,21 & 0,18 & 0,21 & 0,21 \\
\hline Agrícolas & & & & 0,08 & 0,04 & 0,12 & 0,04 & 0,02 \\
\hline
\end{tabular}

Fuente: Elaboración propia en base a información de los censos de población. Para Bogotá la variación es entre 1993 y 2005, para Lima entre 1993 y 2007 y para Santiago entre 1982 y 2002.

Al interpretar la información para Bogotá, se podría decir que su estructura parece ser un poco distinta, debido a que los grupos más aislados son los medios y universitarios, lo cual no calzaría perfectamente de acuerdo a las competencias equivalentes a los grupos educacionales de Lima y Santiago.

Finamente en cuanto a las tendencias, estas son diversas ya que, mientras en Bogotá y Santiago la homogeneidad de los grupos directivos y educación superior aumenta, en Lima disminuye. Sin embargo en donde existe coincidencia es en las tendencias de los sectores medios y bajos de la pirámide socio ocupacional, en donde la variación de los índices nos muestran que los grupos medios comienzan a establecer barrios cada vez más homogéneos y los barrios tradicionalmente obreros comienzan a diversificarse.

\section{Principales hallazgos}

El concepto de neoliberalización híbrida, acuñado por Brenner, Peck y Theodore (2010), nos ayuda a entender las similitudes y diferencias en la instauración de un conjunto de regulaciones que tuvieron como objetivo darle al mercado un papel relevante en la promoción del bienestar, bajo condiciones y contextos particulares en Chile, Colombia y Perú. Si bien se observan ciertas tendencias similares en las tres ciudades, como por ejemplo la precarización del empleo o la segmentación de los espacios residenciales, estos procesos poseen un componente de especificidad que configura territorios con características particulares.

Por ejemplo, en cuanto a la evolución de los mercados laborales urbanos, se pueden analizar tres aspectos principalmente. En primer lugar que el crecimiento económico generado en las tres ciudades no necesariamente ha contribuido en aumentar las tasas de participación laboral, las cuales se mantienen bajas sobre todo en los jóvenes y en los sectores de menores ingresos. Lo segundo es que se están produciendo importantes segmentaciones en el acceso al empleo que se manifiestan en tasas de desempleo muy altas sobre todo en las mujeres, jóvenes y sectores de menores ingresos. Finalmente, lo tercero es que está aumentando crecientemente el empleo desprotegido o de baja calidad, que se manifiesta en la desprotección vía contratos, en la alta rotación laboral y en el aumento de la informalidad, la cual ya era alta sobre todo en los casos de Bogotá y Lima. Sin embargo estas tendencias se manifiestan sobre estructuras laborales diversas, en donde la informalidad juega un rol muy relevante. 
En cuanto a la estructura socioespacial y la distribución de los distintos grupos sociales en las ciudades analizadas, es interesante poner en relieve las desigualdades en las tendencias de los grupos directivos y de los obreros en los casos de Lima y Santiago, los cuales se comportan de una manera distinta. Respecto a las similitudes, la tendencia común a los tres casos sería el proceso de medianización que están experimentando las ciudades, donde los grupos medios y los trabajadores de comercio y servicios, crecen con fuerza, pasando a ser los grupos mayoritarios de la estratificación social. Esta tendencia cuestiona la hipótesis de una dualización urbana generalizada, aunque esconde otros procesos de transformación de la estructura social, asociados a las condiciones del trabajo en las ciudades en proceso de globalización, como ya lo habían adelantado otras investigaciones realizadas para Santiago (De Mattos et al., 2005). A pesar de estas similitudes, lo importante es analizar el comportamiento espacial que tienen estos grupos medios, ya que sería fácil pensar que un crecimiento de esta clase podría favorecer la integración espacial, al actuar como "pegamento" entre los más pobres y los más ricos, sin embargo su tendencia a la privatización en materia de uso de servicios públicos y su tendencia a aglomerarse espacialmente entre sí o junto a la élite y separarse de los más pobres para diferenciarse, ha tendido a jugar en contra de la construcción de un tejido social más equilibrado.

Respecto a las estructuras socioespaciales es posible afirmar que las tres ciudades presentan un marcado sector en donde se concentran los grupos altos de la pirámide social. En el caso de Bogotá es desde el centro histórico hacia el norte, en el caso de Lima en el centro geográfico de cerro a costa y en el caso de Santiago desde el centro histórico hacia el oriente. Sin embargo a pesar de esta similitud, la estructura social de este sector tradicional de altos ingresos es diferente en cada una de las ciudades. En el caso de Bogotá y Santiago existe mayor diversidad social, ya que otros grupos de una clase social más baja también tienen presencia entre ellos, en Lima es mucho más homogéneo y solo se comparte espacio con grupos medios principalmente.

Por el contrario, la localización de los sectores más bajos de la pirámide social que- da relegada a las zonas más periféricas de la ciudad, en el caso de Lima en los bordes externos de los conos, en el caso de Santiago en el poniente y sur de la ciudad formando una especie de media luna, y en el caso de Bogotá principalmente en el sector sur de la ciudad, en todos los casos lejos de los centros y nuevos desarrollos urbanos.

Los hallazgos encontrados no permiten corroborar totalmente la hipótesis. Si bien se comprobó que las reformas estructurales fueron aplicadas en distintos momentos, diferentes intensidades y contextos, y que se están dando procesos importantes de segmentación laboral, los espacios residenciales y las tendencias de segregación residencial se comportan de manera diversa y no existe una tendencia clara que se manifieste con fuerza en las tres ciudades. Tal situación permite reflexionar sobre las particularidades de la producción social del espacio urbano, proceso que responde a lógicas extremadamente complejas que son difíciles de modelizar para establecer patrones generales. En este sentido cada ciudad tiene ciertas particularidades (geografía, estructuras de poder, gobernanza, historia, lógicas sociales, entre otros) que generan combinaciones únicas en un análisis más particular o fino sobre la reproducción del espacio de la ciudad. Con tal aseveración no se pretende desvirtuar las generalizaciones acerca de la evolución de las ciudades, sino que se pretende hacer énfasis en que estas generalizaciones deben ser cuidadosas y que los procesos sociales se manifiestan de una manera mucho más compleja al Ilevarlas al espacio urbano. En este sentido, si bien se pueden encontrar tendencias estructurales, la forma en que estas tendencias se manifiestan en el espacio urbano responden también a determinantes coyunturales, asumiendo, siguiendo a Lefebvre (1974) un cierto grado de autonomía superestructural en la producción del espacio.

A pesar de tal situación y de no corroborar totalmente la hipótesis para los tres casos de estudio, es claro que los procesos que se están dando en las ciudades, a pesar de sus diferencias en las tendencias espaciales y su especifidad, están contribuyendo a mayores amenazas a la convivencia, en donde la segmentación se consolida como fuerte condicionante de la interacción social. Estas 
amenazas se fundan, dentro del contexto que Katzman (2009) ha descrito como específico de este período, que es en el aumento de las expectativas de las personas, ante los favorables escenarios políticos y económicos que se encuentran enfrentando estos países en el contexto actual. Las altas tasas de crecimiento económico, el aumento en la cobertura educacional, la consolidación de la democracia y los discursos políticos, han alimentado las esperanzas de crecientes cantidades de población históricamente relegadas, de mejorar y alcanzar una posición social más igualitaria, con pleno acceso al bienestar y calidad de vida. Sin embargo, la realidad choca con la esperanza de ejercer el pleno derecho de la ciudadanía, dadas las crecientes debilidades que se producen en el vínculo entre los sectores más bajos de la pirámide social y el mercado del trabajo, a lo que se debe sumar el relegamiento y aislamiento espacial.

En este sentido este artículo intenta demostrar que el proceso de reestructuración de las economías metropolitanas, supone el traspaso de los riesgos y las responsabilidades sobre el bienestar a los individuos, y justamente quienes establecen los vínculos más débiles con el mercado del trabajo y quedan relegados en ciertos sectores de la ciudad, son quienes resultan más perjudicados. La disminución de los recursos personales para interactuar en las esferas más importantes de la sociabilidad como son el trabajo y la ciudad, genera un clima en el que los lazos con el resto de la sociedad se rompen y difícilmente podrán volver a ser atados, ya que la segmentación social tiende a reproducirse, dificultando una construcción cohesionada de la ciudad. En palabras de Kaztman y Retamoso (2005: 146): "de corroborarse las tendencias a una distribución espacial cada vez más polarizada de las clases en las ciudades, y de verificarse que en los barrios más desaventajados se activan mecanismos que realimentan el aislamiento social de sus residentes, estaríamos en presencia de procesos que, si no son contrarrestados con eficacia, conducirán ineludiblemente a una reproducción ampliada de las desigualdades, ya extremadamente marcadas, de las que adolecen las grandes ciudades de la región".

Entonces, ante la pregunta sobre la compatibilidad entre ciudad competitiva y ciudad cohesionada, la evidencia para estas tres ciudades parece demostrar que no es posible, al menos bajo esta visión de la competitividad urbana.

\section{Referencias bibliográficas}

ASCHER, F. Los nuevos principios del urbanismo. Barcelona: Editorial Paidós, 2004.

BRENNER, N.; PECK, J. \& THEODORE, $\mathrm{N}$. Variegated neoliberalization: geographies, modolities, pathways. Global Network, 2010, Vol. 10, № 2, p. 182-222.

BUCK, N.; GORDON, I.; HARDING, A. \& TUROK, I. Changing Cities. rethinking Urban, Competitiveness, Cohesion and Governance. New York: Palgrave Macmillan, 2005.

CASTELLS, M. La ciudad y las masas. Madrid: Alianza Editorial, 1986.

COLOMA, F. y ROJAS, P. Evolución del mercado laboral en Chile, reformas y resultados. En: LARRAÍN, F. y VERGARA, R. (editores). La Transformación económica de Chile. Santiago de Chile: Centro de Estudios Públicos, 2001, p. 491-540.

COMISIÓN ECONÓMICA PARA AMÉRICA LATINA Y EL CARIBE (CEPAL). Cohesión Social: Exclusión y sentido de pertenencia en América Latina y el Caribe. Santiago de Chile: CEPAL, 2007a.

COMISIÓN ECONÓMICA PARA AMÉRICA LATINA Y EL CARIBE (CEPAL). Sistema de Indicadores para el seguimiento de la Cohesión Social. Santiago de Chile: CEPAL, 2007b.

DUNCAN, O. \& DUNCAN, B. A Methodological Analysis of Segregation Indexes. American Sociological Review, 1955, $N^{\circ} 20$, p. 210-217.

EDWARDS, S. Crisis y reforma en América Latina: del desconsuelo a la esperanza. Buenos Aires: Emecé editores S.A., 1998.

EDWARDS, S. Veinticinco Años de Inflación y Estabilización en Chile (1973-1998). En: LARRAÍN, F. y VERGARA, R. (editores). La Transformación económica de Chile. San- 
tiago: Centro de Estudios Públicos, 2001, p. 491-540.

ERADYN, A. The impact of globalisation on different social groups: competitiveness, social cohesion and spatial segregation in Istambul. Urban Studies, 2008, Vol. 45, № 8, p. 1.663-1.691.

FAINSTEIN, S. Competitiveness, cohesion and governance: a review of the literature. New Brunswick: Rutgers University, 2001. Disponible en Internet: http://cwis.livjm. ac.uk/cities/conference/sf.pdf

FUENTES, F. y MENESES, F. Subempleo en Chile. Aproximación metodológica y medición. Santiago de Chile: División de Estudios Instituto Nacional de Estadísticas, 1987.

FUENTES, L. Santiago a escala latinoamericana ¿una ciudad competitiva? Santiago de Chile: Tesis para optar al grado de Magíster, Pontificia Universidad Católica de Chile, 2008.

FUENTES, L. Ciudades y sociedades urbanas en transformación. Competitividad, reestructuración y cohesión social en Bogotá, Lima y Santiago. Santiago de Chile: Tesis para optar al grado de doctor, Pontificia Universidad Católica de Chile, 2012.

HAMNETT, C. Why Sassen is wrong: a response to burgers. Urban Studies, 1996, Vol. 33, No 1, p. 107-110.

HARVEY, D. Breve historia del neoliberalismo. Madrid: Ediciones Akal, 2007.

KATZMAN, R. La dimensión espacial de la cohesión social en las grandes ciudades de América Latina. Seminario regional Alcanzando convergencias en la medición de la cohesión social. Santiago de Chile: CEPAL, 2009.

KAZTMAN, R. y RETAMOSO, A. Segregación espacial, empleo y pobreza en Montevideo. Revista de la CEPAL, 2005, No 85, p. 131-148.

KLEIN, N. La doctrina de Shock. El auge del capitalismo del desastre. Buenos Aires: Paidos, 2007.
LEFEBVRE, H. La producción del espacio. Madrid: Capitán Swing, 2012 [1974].

LINK, F. Consecuencias personales de una transformación urbana. La experiencia de segregación en Santiago de Chile. En: NOYOLA, J.; DE MATTOS, C.; ORELLANA, A. (editores). Urbanización en tiempos de crisis. Impactos, desafíos y propuestas. Santiago de Chile: Colección Estudios Urbanos UC - Red de Investigación sobre Áreas metropolitanas de Europa y América Latina RIDEAL, 2013, p. 315-340.

LINK, F. Globalización, vulnerabilidad y riesgo en la nueva configuración socio-territorial del trabajo en el área metropolitana de Santiago. Santiago de Chile: Tesis para optar al grado de Doctor en Arquitectura y Estudios Urbanos, Pontificia Universidad Católica de Chile, 2010.

MASSEY, D. \& DENTON, N. The dimensions of residential segregation. Social Forces, 1988, Vol. 67, №2, p. 281-315.

MELLER, P.; BORDÓN, P.; COCIÑA, M. y RIVERA, E. Mercados Laborales: ¿Soportes o amenazas para la cohesión social latinoamericana? En: TIRONI, E. Redes Estado y Mercados. Soportes de la Cohesión Social Latinoamericana. Santiago de Chile: Uqbar Editores, 2008, p. 227-271.

MINISTERIO DEL TRABAJO Y PROMOCIÓN DEL EMPLEO. Boletín de economía laboral 21. Lima: Ministerio del Trabajo y Promoción del Empleo, 2002.

OCAMPO, J.; PÉREZ, M; TOVAR, C. y LASSO, F. Macroeconomía, ajuste estructural y equidad en Colombia. Bogotá: Departamento Nacional de Planeación - Archivos de Macroeconomía, 1998. Disponible en Internet:

https://www.dnp.gov.co/Portals/0/archivos/ documentos/DEE/Archivos_Economia/79.pdf

OCAMPO, J.; SÁNCHEZ, F. y TOVAR, C. Mercado laboral y distribución del ingreso en Colombia en los años noventa. Revista de la CEPAL, 2000, No 72, p. 53-78.

PEÑA, C. El concepto de Cohesión Social, Debates teóricos y usos políticos. En: TIRONI, E. Redes Estado y Mercados. Soportes de la 
Cohesión Social Latinoamericana. Santiago de Chile: Uqbar Editores, 2008, p. 29-76.

RODRÍGUEZ, J. Segregación residencial socioeconómica: ¿qué es?, ¿cómo se mide?, ¿qué está pasando?, ¿importa? Santiago de Chile: Serie Población y Desarrollo, CEPAL, 2001. Disponible en Internet: http://www.eclac.cl/ publicaciones/xml/8/7888/lcl1576-P.pdf

SABATINI, F.; CÁCERES, G. y CERDA, J. Segregación residencial en las principales ciudades chilenas: tendencias de las tres últimas décadas y posibles cursos de acción. EURE, 2001, Vol. 27, № 82, p. 21-42.

SABATINI, F.; SALCEDO, R.; WORMALD, G. y CÁCERES, G. Tendencias de segregación en las principales ciudades chilenas. Análisis censal 1982-2002. Santiago de Chile: PUC INE, 2008.

SABATINI, F. y SIERRALTA, C. Medición de la segregación residencial. Meandros teóricos y metodológicos y especificidades de la ciudad latinoamericana. En: CUNHA, J. (editor). Novas Metropoles paulistas: populacao, vulnerabilidades e segregacao. Campinas: UNICAMP, 2002, p. 169-195.

SASSEN, S. The global city: New York, London, Tokyo. London: Routledge, 1991.
SECRETARÍA DISTRITAL DE PLANEACIÓN. Evolución del mercado laboral de Bogotá 2001 - 2009. Perspectiva de absorción y calidad del empleo en Bogotá. Bogotá: Alcaldía Mayor de Bogotá DC, 2009.

TIRONI, M. y TIRONI, E. Ciudad, Cohesión y Violencia. La experiencia europea y América Latina. En: TIRONI, E. Redes Estado y Mercados. Soportes de la Cohesión Social Latinoamericana. Santiago de Chile: Uqbar Editores, 2008, p. 323-368.

TUROK, I. The connections between social cohesion and city competitiveness. In: OECD. OECD Competitive cities in the global economy. Paris: OECD, 2006, p. 353-366.

WELLER, J. Panorama de las condiciones de trabajo en América Latina. Nueva Sociedad, 2011, Vol. 232, p. 32-49.

WILLIAMSON, J. A Short History of the Washington Consensus. In: FUNDACION CIDOB. From the Washington Consensus towards a new Global Governance: September 24-25. Barcelona: Fundación CIDOB, 2004, s/p. Disponible en Internet: http:// www.iie.com/publications/papers/williamson0904-2.pdf 